05,11

\title{
Квантование спектра спинонных пар в магнитном поле и формирование бозонов в виде фрагментов 1D-волн зарядовой/спиновой плотности в $\mathrm{SmMnO}_{3+\delta}$
}

\author{
(C) Ф.Н. Буханько, А.Ф. Буханько \\ Донецкий физико-технический институт им. А.А. Галкина НАНУ, \\ Киев, Украина \\ ฯ E-mail: metatem@ukr.net
}

Поступила в Редакцию 14 июня 2021 г.

В окончательной редакции 14 июня 2021 г.

Принята к публикации 18 октября 2021 г.

\begin{abstract}
Исследованы квантовые осцилляции температурных зависимостей намагниченности $\mathrm{SmMnO}_{3+\delta}$ в магнитных полях $H=100$ и $350 \mathrm{Oe}, 1$ и $3.5 \mathrm{kOe}$ в интервале температур $4.2-12 \mathrm{~K}$. Континуум тепловых возбуждений спинонных пар в магнитном поле $H=100$ Ое делится на три перекрывающиеся зоны Ландау с энергиями $E_{n}(n=1,2,3)$ с дробными значениями фактора $v$ заполнения зон. Основной вклад в намагниченность вносит симметричный интенсивный дублет „сверхнамагниченности“, состоящий из двух перекрывающихся пиков вбизи средней температуры возбуждения $T_{\text {spinon }} \cong 8 \mathrm{~K}$. С ростом поля до значения $H=1 \mathrm{kOe}$, спектр возбуждения спинонов трансформируется в широкий синусоподобный пик с вершиной вблизи $T_{\text {spinon }} \cong 8 \mathrm{~K}$, характерный для континуума возбуждений пар спинонов с сильной диперсией в режиме их конфайнмента. В магнитном поле $H=3.5 \mathrm{kOе}$ спектр теплового возбуждения спинонов существенно изменяется: появляется новый тип квантования спектра спинонов в виде формировании в зонах Ландау с $n=1,2$ и 3 особенностей намагниченности в виде узких ступенек (плато), соответствующих целочисленному заполнению спинонами трех зон Ландау с конечной щелью. Исследована эволюция спектра бозонов в виде тепловых возбуждений бесщелевых квазиодномерных волн зарядовой/спиновой плотности в жидкости Латтинжера при температурах ниже $60 \mathrm{~K}$. При температурах вблизи $T=0$ обнаружен непрерывный квантовый фазовый переход квантовой спиновой жидкости в состояние жидкости Латтинжера, индуцированный ростом напряженности магнитного поля. Переход содержит особенности, характерные для формирования 1D-волн зарядовой/спиновой плотности, вызванного конфайнментом спинонных пар, и сопровождается сильным полевым гистерезисом.
\end{abstract}

Ключевые слова: квантовая спиновая жидкость, жидкость Латтинжера, конфайнмент спинонных пар, система спинон - калибровочное поле, квантование Ландау, 1D-волны зарядовой/спиновой плотности, квантовые осцилляции.

DOI: 10.21883/FTT.2022.02.51928.142

\section{1. Введение}

В последнее время большой интерес вызывает модель жидкости Томонаги-Латтинжера, или просто жидкости Латтинжера (ЖЛ). Эта модель хорошо описывает взаимодействие электронов или других фермионов в одномерном проводнике. Такая модель необходима, поскольку обычно используемая модель жидкости Ферми теряет применимость в одномерном случае. Теория ЖЛ описывает низкоэнергетические коллективные возбуждения в одномерном электронном газе как бозоны. Гамильтониан для свободных электронов разделяется по электронам с противоположными (левым-правым) направлениями движения. Среди физических систем, которые описываются этой моделью, выделяют: электроны проводимости в режиме дробного или целочисленного квантового эффекта Холла, 1D-цепочки из полуцелых спинов, описываемых моделью Гейзенберга. Теория ЖЛ хорошо описывает низкоэнергетические свойства широкого класса бесщелевых одномерных взаимодействующих фермионных систем $[1,2]$. Ограничение рассмотре- ния низкими энергиями возбуждения обычно оправдывается линеаризацией спектра физических фермионов вокруг правой и левой точек Ферми $\varepsilon(k) \approx v_{\mathrm{F}}\left( \pm k-k_{\mathrm{F}}\right)$. В рамках этого приближения система может быть строго описана даже при наличии ненулевых взаимодействий между частицами. Теория взаимодействующих фермионов преобразуется в теорию невзаимодействующих бозонов, и все корреляционные функции могут быть точно рассчитаны. Предполагается, что в одномерном случае возбуждения многих частиц в виде волн зарядовой и спиновой плотности, которые подчиняются статистике Бозе, в основном заменяют элементарные возбуждения фермионов. Одной из замечательных особенностей одномерных проводящих систем фермионов, находящихся в состоянии жидкости Латтинжера, является разделение спинов и зарядов. Гамильтониан взаимодействующей системы разделяется на два коммутирующих члена, которые действуют в различных пространствах Гильберта и описывают зарядовые и спиновые степени свободы раздельно. Это необычное состояние одномерных проводящих систем полностью характеризуется скоростями 
волн спиновой и зарядовой плотности (соответственно $v_{s}$ и $\left.v_{c}\right)$ и параметром Латтинжера $K$, который зависит от величины взаимодействия между частицами. Для ненулевых взаимодействий эти скорости отличаются. Коллективная природа собственных колебаний ЖЛ и существование разделения спинов и зарядов четко проявляются в различных функциях динамического отклика. Структурные факторы плотности заряда $S(k, \omega)$ и спина $S^{ \pm}(k, \omega)=S^{x} \pm i S^{y}, S^{z z}(k, \omega)$ измеряют линейный отклик системы частиц с моментом $k$ и энергией $\omega$ на возмущение, которое изменяет плотность зарядов и спинов в системе. Для спиновой ЖЛ динамический структурный фактор плотности заряда $S(k, \omega) \propto \delta\left(\omega-v_{c}|k|\right)$, тогда как динамический структурный фактор плотности спинов $S^{z z}(k, \omega)=1 / 2 S^{ \pm}(k, \omega) \propto \delta\left(\omega-v_{s}|k|\right)$. Наличие $\delta$-образного пика Дирака этих функций отражает тот факт, что зарядовые и спиновые волны являются собственными колебаниями ЖЛ, поэтому каждому значению волнового числа соответствует определенная энергия возбуждения. Этот результат непосредственно связан с линеаризацией спектра фермионов в линейной теории ЖЛ. Следует отметить, что волны зарядовой и спиновой плотности, согласно этой модели, полностью развязаны. Вдали от точек Ферми кривизной физического спектра фермионов $\varepsilon(k)$ невозможно пренебречь: например, искривление спектра фермионов приводит к связыванию волн зарядовой и спиновой плотности [3-5].

В работах [6,7] рассмотрен динамический отклик сильно коррелированных одномерных мотовских диэлектриков со спектральной щелью $M$ с половинным и четвертным уровнем заполнения зоны проводимости. Было получено, что функция динамического отклика зарядов и спинов сильно отличаются в этих двух случаях. Согласно [6], в пределе низких энергий возбуждения обе системы проявляют спин-зарядовое разделение, что позволило точно рассчитать их динамическую спектральную функцию плотности заряда и спина $A\left(\omega, k_{\mathrm{F}}+q\right)$. Было установлено, что в одномерном мотовском диэлектрике с половинным заполнением при температуре $T=0$ операторы возбуждения и аннигиляции ансамбля фермионов с левым и правым направлением движения разделяют их на отдельные зарядовые и спиновые фрагменты в результате бозонизации в виде волн зарядовой и спиновой плотности. В работе представлена серия спектральных функций $A_{R}\left(\omega, k_{\mathrm{F}}+q\right)$, рассчитанная для случаев $v_{s}=0.8 v_{c},-4 M \leq q \leq 4 M$, из которой четко видно существование двух пиков, соответствующих возбуждению волны зарядовой плотности (ВЗП) и волны спиновой плотности (ВСП).

Большая часть спектрального веса сосредоточена в этих особенностях, которые являются прямым проявлением разделения заряд-спин. Пиковая особенность спектральной функции с более низкой (высокой) энергией соответствует ситуации, в которой все фермионы с моментом $q$, участвующие в формировании коллективного континуума возбуждений, являются носителями спина (заряда). Важно отметить, что оба пика имеют существенную ширину. В области низких температур
$T<M$ влияние роста температуры на корреляционную функцию зарядов мало, но спиновая часть спектральной функции может сильно измениться, так как в спектре спинонов щель отсутствует. Было установлено, что спектральная функция одномерного мотовского диэлектрика с четвертным уровнем заполнения не содержит двух четких пиковых особенностей, связанных с раздельным возбуждением волн зарядовой и спиновой плотности. В отличие от половинного заполнения, можно ожидать наличие только одного асимметричного очень широкого дисперсного пика плотности состояний $A\left(\omega, k_{\mathrm{F}}+q\right)$, расположенного вокруг $k_{\mathrm{F}}$.

Большой интерес представляют результаты исследования в работе [6] квазиодномерных свойств цепочек Хаббарда, слабо связанных межцепочечным туннелированием. Для простоты рассмотрен случай, когда взаимодействие между цепочками является дальнодействующим только в направлении, перпендикулярном цепочкам. Подобно случаю связанной ЖЛ, межцепочечное туннелирование $t_{\perp}$ в квазиодномерном мотовском диэлектрике рассматривается как возмущение, которое позволяет расширить область применения предложенного авторами метода исследования на квазиодномерную систему слабосвязанных цепочек. Было получено, что функция Грина $G_{3 \mathrm{D}}(\omega, q, \mathbf{k})$ плотности возбуждений для системы цепочек имеет полюс при $q=k_{\mathrm{F}}$, который соответствует образованию связанного состояния антихолона и спинона с квантовыми числами электрона. При бесконечно малой величине межцепочечного туннелирования $t_{\perp} \neq 0$ это связанное состояние имеет щель с энергией меньшей, чем мотовская щель. При энергиях возбуждения больших, чем мотовская щель, существует континуум возбужденных состояний, подобный полученному для несвязанных цепочек. С ростом $t_{\perp}$ происходит уменьшение щели связанного состояния. Наибольший интерес имеет ситуация, когда эта щель становится очень малой. По мнению авторов, в этом случае поперечные межцепочечные прыжки фермионов являются главным фактором, определяющим формирование замкнутых траекторий в виде петель. Увеличение $t_{\perp}$ сокращает щель связанного состояния до тех пор, пока не формируется поверхность Ферми в виде связанных электронных и дырочных областей, что характерно для металлического состояния. Таким образом, увеличение $t_{\perp}$ в системе слабо связанных цепочек может привести к фазовому переходу диэлектрик-металл квазиодномерного мотовского диэлектрика к металлической жидкости Ферми.

Существование межцепочечной связи в системе антиферромагнитных (АФМ) спиновых цепочек с эффективным спином $S=1 / 2$ сопровождается, согласно многочисленным теоретическим моделям, появлением в них совершенно новых физических свойств, что вызвало значительный интерес экспериментаторов к изучению этих систем [8-14]. В работе [8] сообщается о результатах исследования системы АФМ-цепочек с эффективным значением псевдоспина $S=1 / 2$ в $\mathrm{Yb}_{2} \mathrm{Pt}_{2} \mathrm{~Pb}$ методом рассеяния нейтронов высокого разрешения в сильных магнитных полях. Фундаментальными возбуждениями 
таких цепочек являются спиноны (свободные дробные квазичастицы), которые формируют триплетный континуум возбуждений, когда они объединены в пары. В пределе сильной изинговской анизотропии $\Delta \gg 1$ спиноны можно рассматривать как доменные стенки в АФМ упорядоченном состоянии цепочки. Требование сохранения углового момента проявляется в том, что спиноны всегда создаются парами. Поэтому каждый спинон несет часть $\pm 1 / 2$ изменения углового момента $\Delta S^{z}=0, \pm 1$, которое необходимо в начальной стадии создания доменных стенок в бесконечной цепочке. Так как при движении этих доменных стенок их энергия и угловой момент сохраняются, стенки будут свободно распространяться и переносить при этом квант энергии $E$ и линейный момент $q$, внесенные в систему при их создании. Это приводит к разделению спиновых и электронных степеней свободы, характерному для жидкости Латтинджера. В цепочках спинов с обменным взаимодействием Изинга континуум возбуждений спинонов имеет энергетическую щель, и основное состояние системы спинов упорядочено. Согласно полученным в работе экспериментальным результатам, внешнее магнитное поле закрывает эту щель в $\mathrm{Yb}_{2} \mathrm{Pt}_{2} \mathrm{~Pb}$ и стимулирует переход АФМ спиновых цепочек в состояние подобное спиновой жидкости Латтинжера. Было показано, что межцепочечная связь приводит к ограничению движения спинонов (конфайнмент), которое сопровождается появлением бесщелевой моды собственных продольных колебаний системы спиновых цепочек.

Ранее, в работе [15] нами было найдено квантование Ландау спектра пар низкоэнергетических магнитных возбуждений $Z_{2}$ квантовой спиновой жидкости (КСЖ) в $\mathrm{La}_{0.15} \mathrm{Sm}_{0.85} \mathrm{MnO}_{3+\delta}$ в системе спинон - калибровочное поле в интервале температур 4.2-12 К. Было показано, что формирование непрерывного спектра тепловых возбуждений КСЖ в режиме „слабых магнитных полей“ $H=100,350$ и $1 \mathrm{kOe}$ приводит к квантовым осцилляциям „сверхнамагниченности“ образцов в интервале температур $4.2-12 \mathrm{~K}$ в виде трех узких сдвоенных пиковых особенностей, которые накладываются на экспоненциальное падение намагниченности щелевой КСЖ при $T \rightarrow 0$. Появление необычного спектра низкоэнергетических возбуждений КСЖ объяснено нами в рамках моделей квантования Ландау спектра композитных квазичастиц с дробными значениями фактора $v$ заполнения трех перекрывающихся зон Ландау. В режиме „сильного внешнего магнитного поля“ $H=3.5 \mathrm{kOe}$ обнаружены квантовые осцилляции температурных зависимостей намагниченности несжимаемой жидкости спинонов в виде трех узких ступенек (плато), соответствующих полному заполнению спинонами неперекрывающихся зон Ландау с целочисленными значениями фактора заполнения. В настоящей работе были исследованы особенности температурных зависимостей „сверхнамагниченности“ $\mathrm{SmMnO}_{3+\delta} \quad(\delta \sim 0.1)$, измеренных в полях $H=100$ и $350 \mathrm{Oe}$ и 1 и $3.5 \mathrm{kOe}$ в режиме измерений fieldcooled (FC) в интервале температур $4.2 \leq T \leq 80 \mathrm{~K}$, а также полевые зависимости намагниченности вблизи
$T=0$ в режимах измерений FC и zero-field-cooled (ZFC). Дана интерпретация полученных экспериментальных результатов в рамках существующих моделей.

\section{2. Методика эксперимента}

Образцы самодопированных манганитов $\mathrm{SmMnO}_{3+\delta}$ $(\delta \sim 0.1)$ были получены из высокочистых окислов лантана, самария и электролитического марганца, взятых в стехиометрическом соотношении. Синтезированный порошок прессовали под давлением $10 \mathrm{kbar}$ в диски диаметром 6 и толщиной $1.2 \mathrm{~mm}$ и спекали на воздухе при температуре $1170^{\circ} \mathrm{C}$ в течение $20 \mathrm{~h}$ с последующим снижением температуры со скоростью $70^{\circ} \mathrm{C} / \mathrm{h}$. Полученные таблетки представляли собой однофазную по рентгеновским данным керамику. Рентгенографические исследования проводили при $300 \mathrm{~K}$ на дифрактометре ДРОН-1.5 в излучении $\mathrm{Ni} K_{\alpha 1+\alpha_{2}}$. Симметрия и параметры кристаллической решетки определялись по положению и характеру расщепления рефлексов псевдокубической решетки типа перовскита. Измерения температурных зависимостей намагниченности образцов производились на вибрационном магнитометре VSM EGG, Princeton Applied Research и непромышленном магнитометре в постоянных магнитных полях 100 и $350 \mathrm{Oe}, 1$ и $3.5 \mathrm{kOe}$. Температурные зависимости намагниченности были получены в FC-режиме измерений, при котором образцы предварительно охлаждались в измерительном поле до $4.2 \mathrm{~K}$ с последующим их нагревом до $100 \mathrm{~K}$.

\section{3. Экспериментальные результаты}

Как видно из рис. $1, a$, в магнитном поле $H=100$ Ое в исследованных образцах $\mathrm{SmMnO}_{3+\delta}$ реализуется хорошо известное в литературе основное состояние КСЖ с энергетической щелью $\Delta_{s}$ между основным и возбужденными состояниями. В рамках широко используемых моделей состояния резонирующих валентных связей (RVB) низкоразмерных фрустрированных антиферромагнетиков, для щелевой фазы спиновой жидкости характерно наличие низкоэнергетических магнитных возбуждений основного немагнитного состояния системы спинов, состоящей из синглетных пар спинов ближайших соседей, в виде пар нейтральных квазичастиц со спином $S=1 / 2$ (спинонов) с различными волновыми векторами. Как показано на рис. 1, $a$, тепловое возбуждение пар спинонов происходит в относительно широком интервале температур 4.2-12 K. Возбуждение пар спинонов с коллинеарной ориентацией спинов $S=1 / 2$ в магнитном поле $H=100$ Ое происходит в основном в интервале температур 6-10K в виде дублета из двух широких почти перекрывающихся пиков „сверхнамагниченности“ образцов вблизи средней температуры возбуждения $T_{\text {spinon }} \cong 8 \mathrm{~K}$, что соответствует энергии теплового возбуждения спинонов $E_{\text {spinon }} \cong 0.6 \mathrm{meV}$. При температурах ниже $6 \mathrm{~K}$ наблюдаются два слабых 


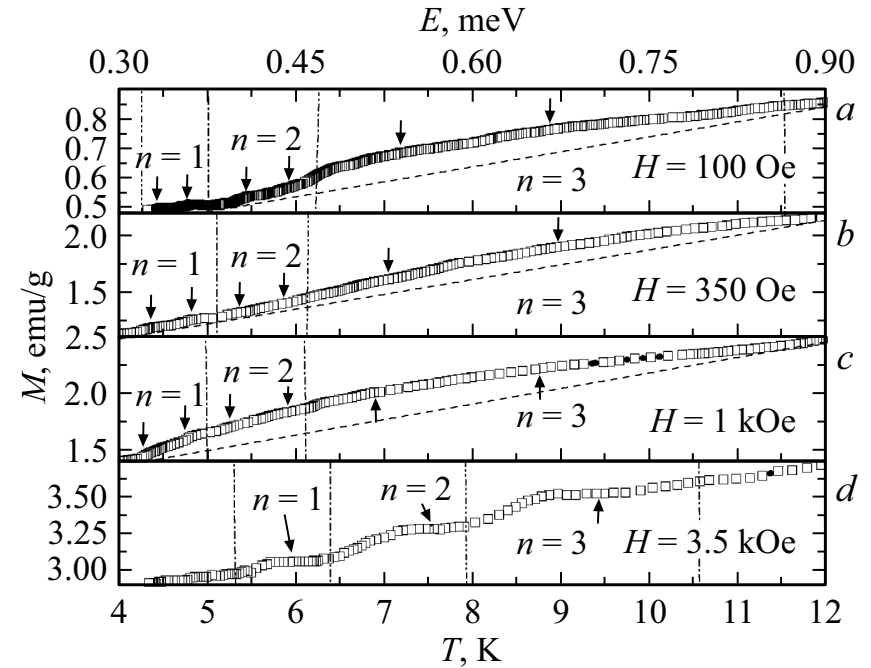

Рис. 1. Квантовые осцилляции температурной зависимости намагниченности $\mathrm{SmMnO}_{3+\delta}$ в магнитном поле $H$. Континуум тепловых возбуждений „сверхнамагниченности“ спинонных пар делится на три перекрывающиеся зоны Ландау $(n=1,2,3) . \quad a) H=100 \mathrm{Oe}$. Осцилляции намагниченности $M(T)$ соответствуют квантованному калибровочным полем спектру низкоэнергетических возбуждений щелевой КСЖ-спинонов в виде периодических сдвоенных пиковых особенностей „сверхнамагниченности“, которые накладываются на падение $M(T)$ при $T \rightarrow 0 . b)$ Формирование интенсивного асимметричного пика „сверхнамагниченности“ $\mathrm{SmMnO}_{3+\delta}$ в магнитном поле $H=350 \mathrm{Oe}$ с вершиной вблизи $T_{\text {spinon }} \cong 8 \mathrm{~K}$. Наблюдается уменьшение относительного вклада возбуждений спинонных пар в узких зонах Ландау с энергиями $E_{1,2}<\Delta_{s} \approx 0.4 \mathrm{meV}$ в „сверхнамагниченность“ образца. c) Формирование интенсивного синусоподобного пика „сверхнамагниченности“ $\mathrm{SmMnO}_{3+\delta}$ в магнитном поле $H=1 \mathrm{kOe}$ с вершиной вблизи $T_{\text {spinon }} \cong 8 \mathrm{~K}$. Этот результат вызван дальнейшим ростом „гигантских“ флуктуаций, возникающих в основном состоянии КСЖ с ростом $H$, что приводит к дальнейшему размазыванию расширенного спектра низкоэнергетических возбуждений спинонов в зоне Ландау с номером $n=3$. Наблюдается дальнейшее уменьшение относительного вклада возбуждений спинонных пар в узких зонах Ландау с номерами $n=1$ и 2 в „сверхнамагниченность“ образца. $d) H=3.5 \mathrm{kOe}$.

дублета намагниченности спинонных пар, с энергией возбуждения $E_{\text {spinon }}$ меньшей, чем низкоэнергетическая щель $\Delta_{s} \approx 0.4 \mathrm{meV}$ в спектре возбуждения спинонов, существующая в 2D-системе спинов между немагнитными синглетными состояниями спинов в основном RVB-состоянии КСЖ и возбужденным магнитным состоянием КСЖ в виде спинонов с коллинеарной ориентацией спинов $S=1 / 2$. Дублетное возбуждение спинонных пар вблизи $T_{\text {spinon }}$ происходит в интервале температур, который перекрывается с температурами развязывания пар 2D-вихрей сверхпроводящей жидкости при температуре вблизи температуры $T_{\mathrm{KT}} \cong 12 \mathrm{~K}$ топологического фазового перехода Костерлитца-Таулесса развязывания 2D-пар вихрь-антивихрь в когерентном сверхпроводящем состоянии, что свидетельствует о практическом совпадении энергий их возбуждения. Согласно рис. $1, a$, в магнитном поле $H=100 \mathrm{Oe}$ в образце $\mathrm{SmMnO}_{3+\delta}$ в интервале температур $4.2-9.5 \mathrm{~K}$ наблюдается непрерывный спектр тепловых возбуждений пар спинонов в виде периодических сдвоенных узких пиковых особенностей температурных зависимостей сверхнамагниченности вблизи средних температур $T_{1} \cong 4.6 \mathrm{~K}, T_{2} \cong 5.6 \mathrm{~K}$ и $T_{3} \cong 8 \mathrm{~K}$, которые накладываются на квазилинейное падение намагниченности образца при $T \rightarrow 0$.

Это позволило разделить непрерывный континуум тепловых возбуждений намагниченности на узкие зоны $n=1,2$ и 3 с характерными для каждой из них особенностями намагниченности, что можно объяснить квантованием спектра спинонов с дробными значениями фактора $v$ заполнения трех перекрывающихся зон Ландау в режиме слабого внешнего магнитного поля. Как видно из рис. $1, b$ и $c$, с ростом напряженности внешнего магнитного поля до значения $H=1 \mathrm{kOe}$ происходит сильное размазывание спектра спинонных пар в узких зонах Ландау с $n=1,2$ и 3, что приводит к формированию симметричного одиночного синусоподобного пика „сверхнамагниченности“ $\mathrm{SmMnO}_{3+\delta}$ в относительно широком интервале температур $4.2-12 \mathrm{~K}$ с вершиной вблизи $T_{\text {spinon }} \cong 8 \mathrm{~K}$. Этот результат свидетельствует о появлении „гигантских“ флуктуаций, возникающих в основном состоянии КСЖ с ростом напряженности магнитного поля, что приводит к размазыванию спектра низкоэнергетических спинонных возбуждений. Таким образом, спектр низкоэнергетических возбуждений спинонных пар в $\mathrm{SmMnO}_{3+\delta}$ в слабых магнитных полях $H \leq 1 \mathrm{kOe}$ существенно отличается от исследованного в [15] спектра возбуждения спинонов в близком по составе манганите $\mathrm{La}_{0.15} \mathrm{Sm}_{0.85} \mathrm{MnO}_{3+\delta}$ - как по форме, так и по зависимости от слабых внешних воздействий (магнитное поле, температура). Отличительной особенностью температурных зависимостей „сверхнамагниченности“ $M(T)$, полученных с ростом внешнего магнитного поля до $3.5 \mathrm{kOe}$, является появление четко выраженных ступенчатых осцилляций намагниченности газа спинонов. Как показано на рис. $1, d$, характерной особенностью осциллирующей в интервале температур $4.2-11 \mathrm{~K}$ сверхнамагниченности является появление в зонах $n=1,2$ и 3 зависимостей „сверхнамагниченности“ 2D-газа спинонов в виде трех узких ступенек (плато) эквидистантных узких пороговых особенностей шириной $\Delta T \cong 1-2 \mathrm{~K}$. С ростом $T$ высота порогов и ширина ступенек (плато) растут. Новые квантовые осцилляции зависимостей $M(T)$ соответствуют целочисленному заполнению спинонами трех уровней Ландау с конечной щелью в режиме сильного внешнего магнитного поля, как было показано нами в [15]. Таким образом, даже относительно небольшое увеличение напряженности внешнего магнитного до поля $H=3.5 \mathrm{kOe}$ привело к переходу от непрерывного спектра парных возбуждений спинонов к дискретному.

Формирование фрагментов зарядов/спинов в виде флуктуирующих ВЗП/ВСП состояний ЖЛ обнаружено 


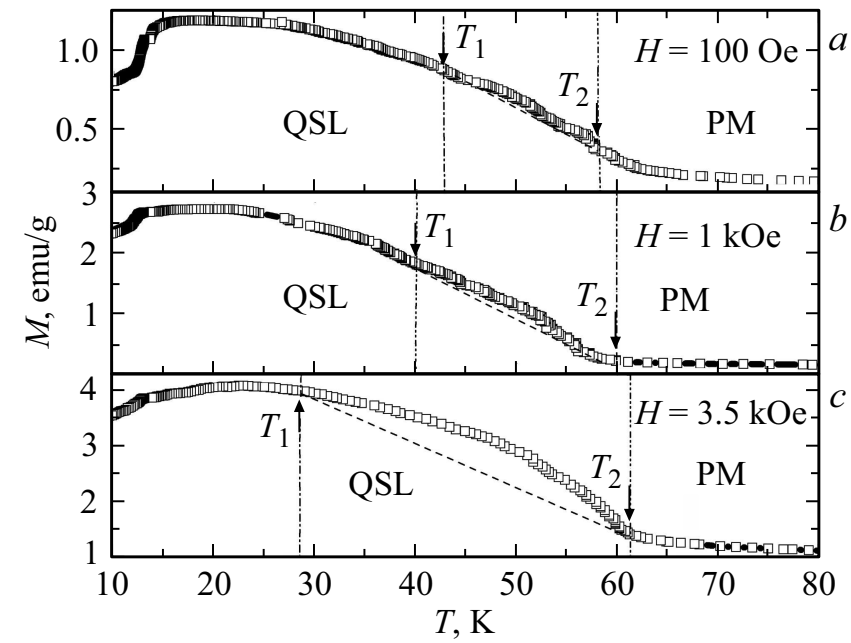

Pис. 2. Температурная зависимость намагниченности $M(T)$ в магнитном поле $H$ в интервале температур $10-80 \mathrm{~K}$. a) Разделение спинов и зарядов в $\mathrm{SmMnO}_{3+\delta}$ в магнитном поле $H=100$ Ое при температурах ниже $60 \mathrm{~K}$. В интервале температур $T_{1}<T<T_{2}$ шириной порядка $15 \mathrm{~K}$ формируется симметричная особенность температурной зависимости намагниченности образца в виде двух пиков „сверхнамагниченности“ равной интенсивности вблизи температур 48 и $56 \mathrm{~K}$, которые вызваны тепловым возбуждением жидкости Латтинжера флуктуирующих квазиодномерных фрагментов раздельных волн зарядовой и спиновой плотности. b) $H=1 \mathrm{kOe}$. c) $H=3.5 \mathrm{kOe}$. (QSL - квантовая спиновая жидкость, РМ - парамагнитное состояние.)

нами в FC режиме измерений температурных зависимостей намагниченности в полях $H=100 \mathrm{Oe}, 1$ и $3.5 \mathrm{kOe}$ при температурах ниже $60 \mathrm{~K}$ (рис. 2-5). Как видно из рис. $2, a$ и 3 , в магнитном поле $H=100 \mathrm{Oе}$ в интервале температур $T_{1}<T<T_{2}$ шириной порядка $15 \mathrm{~K}$ формируется симметричная двухпиковая особенность „сверхнамагниченности“ образца в виде двух четко выраженных пиков намагниченности $M(T)$ равной интенсивности вблизи температур 48 и $56 \mathrm{~K}$, которые вызваны тепловым возбуждением флуктуирующих квазиодномерных фрагментов волн зарядовой и спиновой плотности. Подобные пиковые особенности намагниченности $M(T)$ были исследованы нами ранее в работе [15] в различных магнитных полях $H$ и режимах измерения. Согласно этой работе, появление двух пиковых особенностей различной ширины и интенсивности при температурах ниже $60 \mathrm{~K}$ можно объяснить существованием малой псевдощели $\Delta_{e}$ в спектре электронов, характерной для слабого Мотовского диэлектрика со спинонной поверхностью Ферми. С ростом напряженности магнитного поля до значения $H=1 \mathrm{kOe}$ картина резко изменилась (рис. 2, $b$ и 4).

Как показано на рис. 4, вблизи температуры $50 \mathrm{~K}$ формируется ассиметричный пик „сверхнамагниченности“, шириной $16 \mathrm{~K}$, который мы связываем с тепловым возбуждением в образце флуктуирующего связанного состояния волн зарядовой и спиновой плотности в ЖЛ.
Дальнейшее увеличение напряженности внешнего магнитного поля до значения $H=3.5 \mathrm{kOe}$ к еще более значительному изменению низкоэнергетического теплового возбуждения ЖЛ: вблизи $T=50 \mathrm{~K}$ формируется

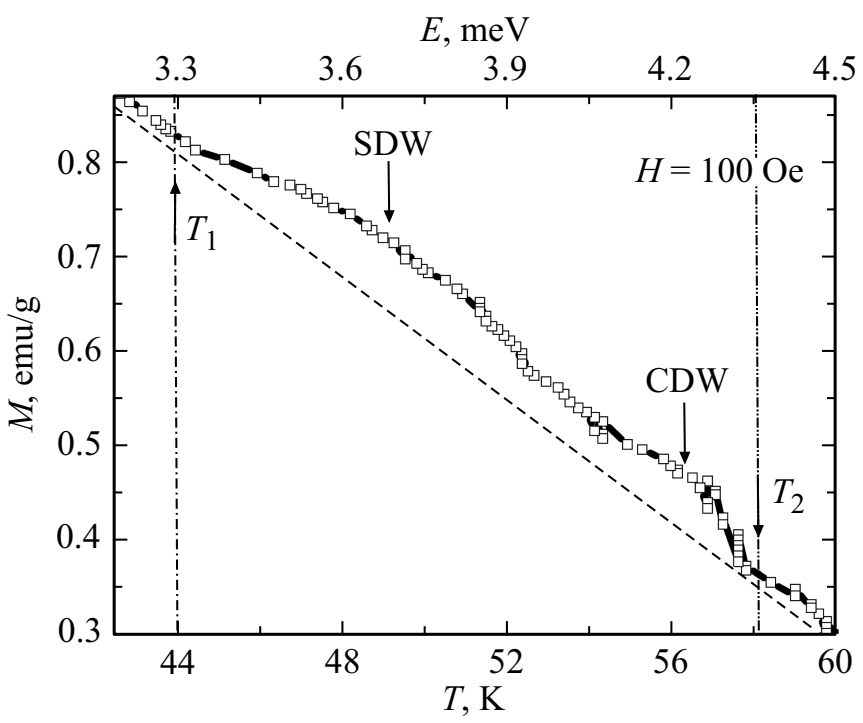

Рис. 3. Формирование бозонов в $\mathrm{SmMnO}_{3+\delta}$ в магнитном поле $H=100$ Ое при температурах ниже $60 \mathrm{~K}$. В интервале температур $T_{1}<T<T_{2}$ шириной около $15 \mathrm{~K}$ формируется симметричная особенность температурной зависимости намагниченности образца в виде двух пиков „сверхнамагниченности“ равной интенсивности вблизи температур 48 и $56 \mathrm{~K}$, которые вызваны тепловым возбуждением жидкости Латтинжера флуктуирующих квазиодномерных фрагментов волн зарядовой $(\mathrm{CDW})$ и спиновой $(\mathrm{SDW})$ плотности.

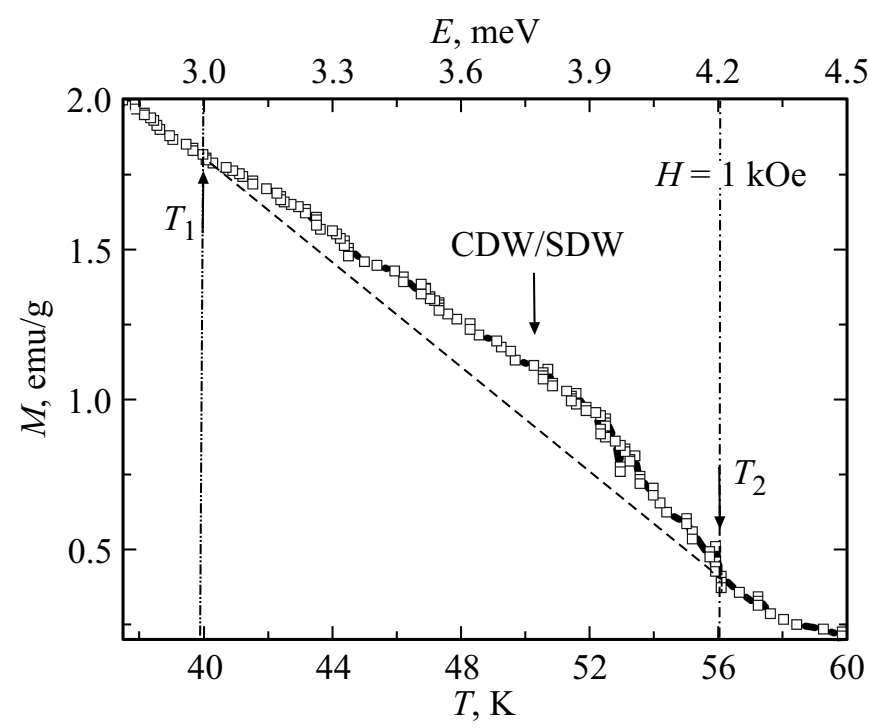

Pис. 4. С ростом напряженности магнитного поля до значения $H=1 \mathrm{kOe}$ на температурной зависимости намагниченности $M(T)$ вблизи температуры $50 \mathrm{~K}$ формируется ассиметричный пик „сверхнамагниченности“ шириной $16 \mathrm{~K}$, который мы связываем с тепловым возбуждением в образце флуктуирующего связанного состояния жидкости Латтинжера. 


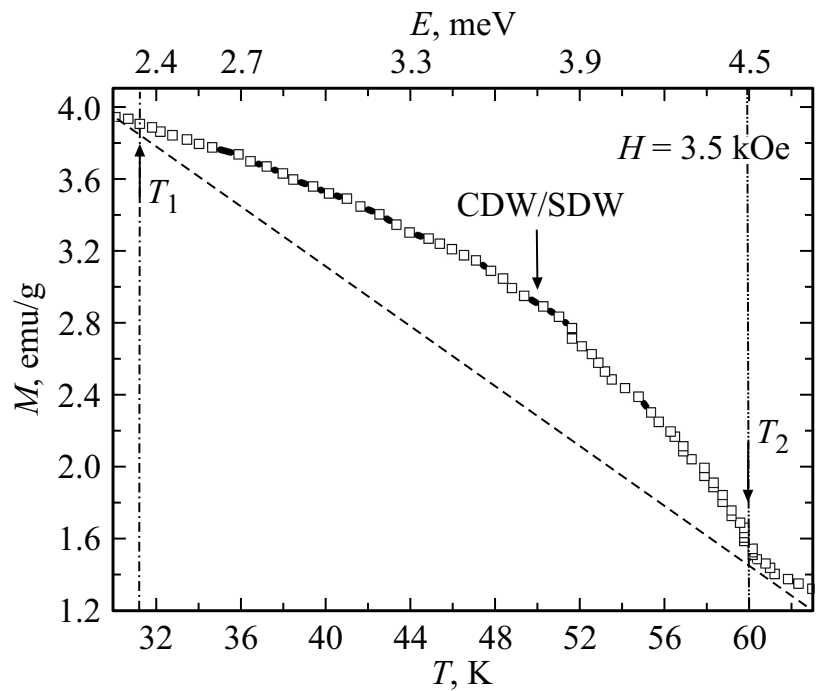

Рис. 5. Увеличение напряженности внешнего магнитного поля до значения $H=3.5 \mathrm{kOe}$ приводит к существенному изменению низкоэнергетического теплового возбуждения жидкости Латтинжера: вблизи $50 \mathrm{~K}$ формируется значительно более широкий синусоподобный пик „сверхнамагниченности“.

значительно более широкий синусоподобный пик „сверхнамагниченности“ (рис. 2, с и 5).

В настоящей работе был найден также конфайнмент низкоэнергетических тепловых возбуждений спинонов в $\mathrm{SmMnO}_{3+\delta}$ в виде возбуждений типа нанофрагментов 1D-волн зарядовой и спиновой плотности, характерных для низкоэнергетических возбуждений металлической одномерной квантовой ЖЛ. Согласно полевым зависимостям намагниченности $M(H)$, измеренных в различных режимах при температурах вблизи $T=0$ (рис. 6,7), с ростом напряженности внешнего магнитного поля происходит квантовый фазовый переход второго рода КСЖ в состояние ЖЛ, индуцированный ростом напряженности внешнего магнитного поля. В результате этого перехода вблизи значения $H=0$ формируются пиковые особенности „сверхнамагниченности“ с различной степенью их перекрытия, характерные для возбуждения бесщелевых развязанных волн зарядовой и спиновой плотности в ЖЛ. На рис. 6 и 7 представлены полевые зависимости намагниченности $\mathrm{SmMnO}_{3+\delta}$ вблизи $T=0$ в FC- и ZFC-режимах измерений в виде изотерм 1 и 2 перемагничивания образцов с различным направлением роста напряженности внешнего магнитного поля. Как видно из рисунков, в процессе перемагничивания в двух режимах измерения формируются две пиковые особенности намагниченноси $M(H)$ вблизи $H=0$ в интервале магнитных полей $\pm 500 \mathrm{Oe}$. Согласно [6], в пределе низких энергий возбуждения одномерного Мотовского диэлектрика происходит спинзарядовое разделение, что позволило точно рассчитать их динамическую спектральную функцию плотности заряда и спина $A\left(\omega, k_{\mathrm{F}}+q\right)$. Было установлено, что в одномерном мотовском диэлектрике при температуре
$T=0$ операторы возбуждения и аннигиляции ансамбля фермионов с левым и правым направлением движения разделяют их на отдельные зарядовые и спиновые области в результате бозонизации в виде фрагментов разделенных одномерных волн зарядовой и спиновой плотности, несоразмерных с кристаллической решеткой.

Мы считаем, что появление двух необычных пиковых особенностей изотерм 1 и 2 перемагничивания $\mathrm{SmMnO}_{3+\delta}$ вблизи нулевого магнитного поля являются

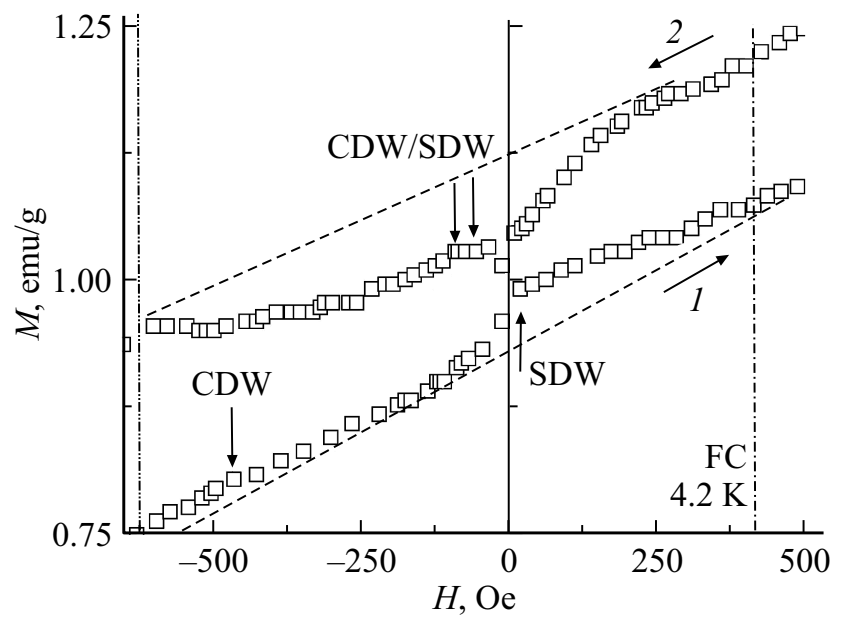

Рис. 6. Полевые зависимости намагниченности $\mathrm{SmMnO}_{3+\delta}$ при $T=4.2 \mathrm{~K}$ в FC-режиме измерений в виде изотерм 1 и 2 перемагничивания образцов с различным направлением роста напряженности внешнего магнитного поля. В процессе перемагничивания образца в FC-режиме измерения формируются две пиковые особенности намагниченности вблизи $H=0$ в интервале магнитных полей $\pm 500 \mathrm{Oe}$. (FC - field-cooled.)

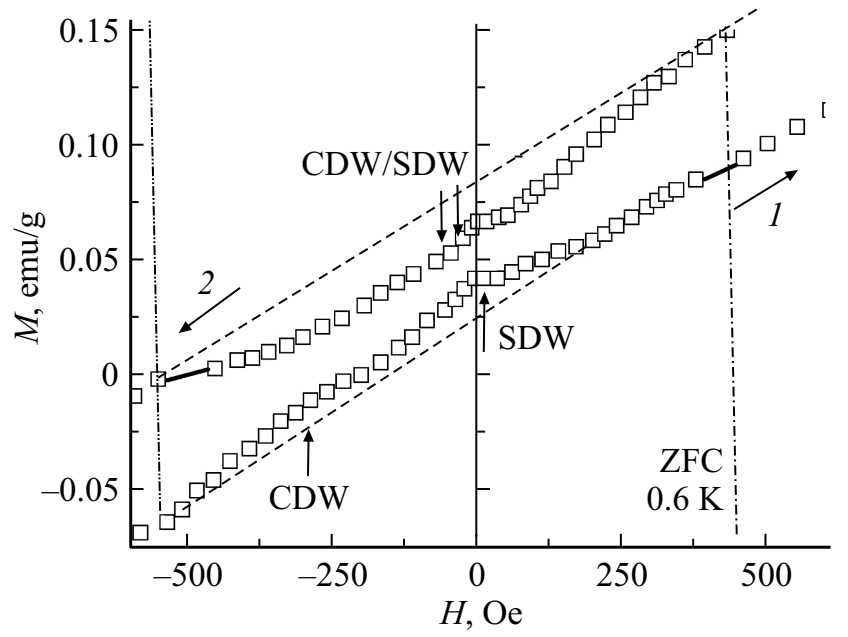

Рис. 7. Полевые зависимости намагниченности $\mathrm{SmMnO}_{3+\delta}$ при $T=0.6 \mathrm{~K}$ в ZFC-режиме измерений в виде изотерм 1 и 2 перемагничивания образцов с различным направлением роста напряженности внешнего магнитного поля. Как видно из рисунка, в процессе перемагничивания образца в ZFC-режиме измерения формируются две пиковые особенности намагниченности вблизи $H=0$ в интервале магнитных полей $\pm 500 \mathrm{Oe}$. (ZFC - zero-field-cooled.) 
естественным следствием спин-зарядового разделения, характерным для хорошо исследованного ранее теоретически одномерного мотовского диэлектрика в состоянии квантовой ЖЛ $[6,7]$.

Обращает на себя внимание отличие двух пиковых особенностей изотерм 1 и 2 перемагничивания вблизи $H=0$, зависящее от направления роста напряженности внешнего магнитного поля. Как видно из рис. 6 и 7, с ростом магнитного поля в положительном направлении (изотерма 1) реализуются раздельные ВЗП и ВСП состояния, не связанные между собою. В то же время, при изменении знака роста поля на противоположный (изотерма 2) мы наблюдаем вблизи $H=0$ связанные между собою ВЗП/ВСП состояния.

Мы считаем, что такое поведение намагниченности в области низких температур вызвано тем, что поперечная компонента внешнего магнитного поля закрывает спинонную щель $\Delta_{s}$ в $\mathrm{SmMnO}_{3+\delta}$ и стимулирует переход системы слабо связанных обменным взаимодействием зигзагообразных АФМ спиновых цепочек в состояние, подобное металлической ЖЛ. Характерной особенностью этого состояния является пространственное разделение зарядовых и спиновых возбуждений. В этом квазиодномерном состоянии доминируют возбуждения многих частиц в виде 1D-волн зарядовой и спиновой плотности, которые подчиняются статистике Бозе, в основном заменяют элементарные возбуждения в виде спинонных пар.

Представляет интерес результат, что в изотермах 1 формируются развязанные волны зарядовой/спиновой плотности, тогда как в изотермах 2 вблизи нулевого магнитного поля возникает связанное 1D BЗП/ВСП коллективное возбуждение квантовой спиновой жидкости. Возбуждение подобных коллективных 1D-состояний во внешнем магнитном поле наблюдалось ранее в системах, слабо связа-ных обменом гейзенберговских и изинговских АФМ спиновых цепочек с различной конфигурацией и степенью анизотропии. Согласно этим работам, даже слабый рост поперечной компоненты внешнего магнитного поля может привести к дальнейшему конфайнменту спинонных пар, которое сопровождается появлением бесщелевых мод собственных продольных колебаний системы спиновых цепочек.

\section{4. Обсуждение экспериментальных результатов}

\section{1. Квантование Ландау спектра спинонов калибровочным полем в щелевой $Z_{2}$ квантовой спиновой жидкости с магнитным потоком}

Ранее в работе [15] нами дано подробное качественное объяснение эволюции квантовых осцилляций „сверхнамагниченности“, впервые измеренных в самодопированном манганите $\mathrm{La}_{0.15} \mathrm{Sm}_{0.85} \mathrm{MnO}_{3+\delta}$ в интервале температур 4.2-100 K, в рамках существующих моделей среднего поля квантования Ландау спектра низкоэнергетических возбуждений $Z_{2}$ квантовой спиновой жидкости с потоком [16-34]. В работе [17] в рамках модели Хаббарда было рассмотрено квантование спинонов в КСЖ со спинонной поверхностью Ферми, находящейся в состоянии, близком к мотовскому переходу диэлектрик-металл. Построен эффективный спиновый гамильтониан в треугольной решетке для случая четырехспинового обменного взаимодействия в присутствии внешнего магнитного поля. Первый член гамильтониана соответствует обмену между парами ближайших спинов с энергией $J_{2}$, тогда как второй член соответствует кольцевому обменному взаимодействию для четверки ближайших спинов с энергией $J_{4}$. В присутствии внешнего магнитного поля в спиновом гамильтониане появляется дополнительный член, который является суммой трехспиновых обменных взаимодействий, связанных с движением спинов в отдельных треугольниках. Этот член пропорционален малому потоку внешнего магнитного поля $\Phi_{\Delta}^{\text {ext }} \ll 1$ через треугольник с постоянной связи $J_{3}$. Показано, что внешнее магнитное поле линейно связано с киральностью спинов в треугольной решетке. Киральность спинов соответствует потоку внутреннего калибровочного поля в рамках теории калибровочного поля, часто используемой для описания спиновой жидкости. Согласно предложенной модели, внешнее магнитное поле индуцирует в системе спинов с четырехспиновым обменным взаимодействием статический внутренний поток. Количественная оценка эффекта позволила установить, что величина возникающего при этом магнитного орбитального поля, действующего на спиноны, сравнима и даже превышает величину внешнего магнитного поля. Утверждается, что в связи с тем, что жесткость внутреннего калибровочного поля очень мала, однородное состояние системы спинон-калибровочное поле неустойчиво в области низких температур из-за сильного квантования Ландау энергетического спектра спинонов. Эта неустойчивость напоминает ситуацию в металлах в режиме сильного магнитного поля, но согласно произведенной в работе количественной оценке, диапазон температур и полей существования неустойчивости однородного состояния в системе спинон-калибровочное поле значительно шире. Отклик системы спинон-калибровочное поле изменяется драматически при низких температурах: квантование Ландау спинонов в статическом внутреннем поле при температурах ниже критического значения не размазано температурой. Было установлено, что для квантовой спиновой жидкости в $k-(\mathrm{ET})_{2} \mathrm{Cu}_{2}(\mathrm{CN})_{3}$ однородное состояние с непрерывно изменяющимся внутренним полем становится неустойчивым при температурах ниже несколько градусов Кельвина при умеренных значениях внешнего магнитного поля. Подобное явление хорошо исследовано ранее для магнитных осцилляций в обычных металлах. Режим нестабильности однородного состояния в системе спинон-калибровочное поле значительно шире, чем в обычных металлах, потому что жесткость внутреннего калибровочного магнитного 
поля значительно меньше, чем внешнего поля. Грубо говоря, в системе спинон-калибровочное поле спинонные состояния с целочисленным заполнением уровней Ландау более устойчивы, чем состояния с непрерывно меняющимся заполнением. Поэтому энергетически выгодно внутреннему калибровочному полю регулировать свою дискретность чтобы достигнуть этого. Такая неустойчивость дает возможность прямого исследования свойств спинонной поверхности Ферми, используя результаты экспериментального исследования осцилляций намагниченности образца. В работе подробно обсуждается механизм квантования Ландау спектра системы спинон-калибровочное поле с ростом напряженности внешнего магнитного поля и температуры образца. Вначале было рассмотрено неоднородное состояние системы спинонов при температуре $T=0$. В нулевом внешнем магнитном поле $\left(\Phi^{\mathrm{ext}}=0\right)$ при слабом кольцевом обмене поток через каждый треугольник $\Phi_{\Delta}=\pi / 2$, тогда как в случае сильного кольцевого обмена оптимальным является состояние с нулевым потоком. Было получено, что при $\Phi^{\mathrm{ext}}=0$ состояния с потоком имеют большую абсолютную восприимчивость $\left|\chi_{\Phi}\right| \leq\left|\chi_{0}\right|\left(1+c \Phi_{\Delta}^{2}\right)$, чем состояние без потока. При малом внешнем потоке $\Phi^{\mathrm{ext}} \neq 0$ в системе спинов с треугольной решеткой генерируется статический поток внутреннего калибровочного поля $\Phi^{\text {int }} \equiv \gamma \Phi^{\mathrm{ext}}$, где $\gamma \gg 1$. Таким образом, эффективное орбитальное поле, действующее на спиноны, сравнимо по величине с приложенным магнитным полем. В рамках модели среднего поля произведен численный расчет зависимости магнитной восприимчивости $\left|\chi_{\Phi}\right|$ системы спинон-калибровочное поле от фактора заполнения уровней Ландау $v$. В присутствии статического внутреннего потока $\Phi_{\Delta}^{\text {int }}$ в элементарных треугольниках спектр спинонов состоит из зон Ландау. Для любой из двух ориентаций спинов фактор заполнения уровней Ландау $v=\pi /\left(2 \Phi_{\Delta}^{\text {int }}\right)$; при этом состояния внутреннего магнитного потока становятся особенными, если $v$ принимает целочисленные значения. В рамках модели среднего поля было получено, что вблизи целочисленных значений фактора заполнения уровней Ландау магнитная восприимчивость системы спинон-калибровочное поле $\left|\chi_{\Phi}\right|=\left|\chi_{0}\right|\left(1+c \Phi_{\Delta}^{2}\right)-\Xi_{\Phi}^{\text {osc }}$ (где $\Xi_{\Phi}^{\text {osc }}$ - осциллирующая составляющая магнитной восприимчивости) имеет форму серии острых пиков, направленных вверх, размеры которых быстро уменьшаются с ростом $v$, так как осциллирующая компонента магнитной восприимчивости $\Xi_{\Phi}^{\text {osc }} \propto v^{-2}(v-k)(k+1-v)$. Квантовые осцилляции $\left|\chi_{\Phi}\right|$ возникают вследствие дискретности спинонных уровней Ландау в виде серии пошаговых переходов первого рода системы спинон-калибровочное поле через эти состояния. В режиме измерений магнитной восприимчивости системы, при непрерывном изменении напряженности внешнего магнитного поля внутренний поток $\Phi^{\text {int }}$ будет ступенчато проходить через дискретный набор значений, соответствующих целочисленному заполнению спинонами уровней Ландау. В этом случае спектр спинонов имеет щель соответствующую циклотронной частоте $\hbar \omega_{c} \approx 3.57 t_{\text {spinon }} / v$.
Это состояние спинонов в сущности является хорошо известным киральным состоянием спиновой жидкости с внутренним калибровочным полем [20]. Осциллирующую составляющую энергии спинонов $\varepsilon^{\text {osc }}=12 t_{\text {spinon }} \Xi_{\Phi}^{\text {osc }}$ $\left(\right.$ где $\left.t_{\text {spinon }}=2 g_{2} J_{2}\left|\chi_{0}\right|+32 g_{4} J_{4}\left|\chi_{0}\right|^{3}\right)$ можно интерпретировать как осциллирующую часть эффективной спинонной кинетической энергии. Конечная температура смазывает эффекты дискретности уровней Ландау; при этом неустойчивость потока с непрерывно изменяющимся значеним $\Phi_{\text {int }}$ становится меньше. Появление серий плато намагниченности фрустрированных гейзенберговских АФМ и киральной спиновой жидкости, вызванное внешним магнитным полем, тесно связано с квантованием Ландау спектра спинонов калибровочным полем Черна-Саймонса [35-37].

Согласно [20], основное состояние бесщелевой фазы $Z_{2}$ КСЖ с потоком описывается гамильтонианом $H_{\text {mean }}$, который эквивалентен гамильтониану, используемому для описания прыжков электронов между узлами кристаллической решетки с „магнитным“ потоком $\pi$ через площадку. Поверхность Ферми для системы фермионов в этом состоянии содержит при половинном заполнении зоны проводимости особые точки при $\left(k_{x}, k_{y}\right)=(0,0)$ и $(0, \pi / a)$. Состоянию спиновой жидкости с потоком соответствуют бесщелевые возбуждения спинов через особые точки поверхности Ферми. В рамках модели среднего поля возможно также существование фазы $Z_{2}$ щелевой спиновой жидкости с топологическим параметром порядка $\chi_{i, j}$, который генерирует поток. Фермионы, описываемые гамильтонианом $H_{\text {mean }}$, ведут себя так, как если бы они двигались в магнитном поле. Когда поток строго соразмерен с плотностью фермионов (что соответствует одному фермиону на узел), целое число уровней (полос) Ландау будет полностью заполнено. В этом случае газ фермионов становится несжимаемым, так как возникает конечная щель между уровнями Ландау. При этом флуктуации плотности фермионов отсутствуют. Возникновение этого квантованного состояния фермионов сопровождается спонтанным нарушением симметрии инверсии времени и четности. Соответствующие состояния системы спинов названы киральными спиновыми состояниями. Связь между ансамблем фермионов и калибровочным полем $a_{\mu}$ идентична связи между электронами и электромагнитным полем. Таким образом, можно ожидать, что для системы фермионов, управляемых калибровочным полем, характерно явление, подобное эффекту Холла. „Эффект Холла“ в данном случае состоит в том, что „электрическая“ компонента калибровочного поля $a_{\mu}$ индуцирует ток фермионов в направлении поперечном направлению „магнитной“ компоненты $b$ калибровочного поля $a_{\mu}$. Существует мнение, что холловская проводимость заполненной зоны фермионов квантуется полем $b$ как целое число, умноженное на $1 / 2 \pi$. Можно изменять плотность управляемых фермионов в киральной спиновой жидкости без создания фермионов в зоне проводимости или дырок в валентной зоне. Для этого достаточно медленного изменения направления 
(поворота) „магнитного“ потока $\Phi=\int d^{2} x b$ калибровочного поля $a_{\mu}$, вызванного внешними воздействиями. Вращение потока индуцирует круговое „электрическое“ поле $e_{\theta}$, которое, в свою очередь, генерирует ток управляемых фермионов в радиальном направлении. Таким образом происходит накопление заряда вблизи „магнитного“ потока Ф. Было показано, что полное число индуцированных изменением потока Ф фермионов равно $N=-\sigma_{x y} \Phi=-\Phi / \pi$. Согласно полученным в данной работе экспериментальным результатам, таким воздействием, меняющим поток $\Phi$ калибровочного поля $a_{\mu}$, может быть изменение внешнего магнитного поля. Сильное отличие квантования спектра спинонов в $\mathrm{SmMnO}_{3+\delta}$ и $\mathrm{La}_{0.15} \mathrm{Sm}_{0.85} \mathrm{MnO}_{3+\delta}$, по-видимому, вызвано разницей энергий межцепочечного обменного взаимодействия.

\section{2. Формирование бозонов в виде 1D-волн зарядовой/спиновой плотности в системах АФМ спиновых цепочек, вызванное конфайнментом спинонных пар}

В работе [8] методом неупругого рассеяния нейтронов высокого разрешения в сильных магнитных полях был исследован механизм формирования бесщелевой моды собственных продольных колебаний системы АФМ спиновых цепочек в $\mathrm{Yb}_{2} \mathrm{Pt}_{2} \mathrm{~Pb}$. Ранее проведенные эксперименты по рассеиванию нейтронов показали, что $4 f$-орбитальное перекрытие приводит к необычным магнитным свойствам металла $\mathrm{Yb}_{2} \mathrm{Pt}_{2} \mathrm{~Pb}$, в котором ортогональные пары ионов $\mathrm{Yb}$ с псевдоспином $S=1 / 2$ расположены в тетрагональных $a b$-плоскостях. Было установлено, что в нулевом магнитном поле возбуждениями магнитной системы спиновых цепочек являются низкоэнергетические спиноны, которые образуют сильно дисперсный континуум возбуждений с шириной, значительно превышающей энергетическую щель, при волновом векторе $\mathbf{q}_{\mathrm{L}}$, направленном вдоль цепочек. Границы двуспинонного континуума возбуждений определяются экстремальными значениями энергии $E$ и линейного момента $q$ пары спинонов, которые сохраняются одной частицей и одной дыркой со спином $S^{z}= \pm 1 / 2$ соответственно. В нулевом магнитном поле химический потенциал $v=0$ располагается посредине энергетической щели $\Delta_{\mathrm{S}}$ спинонов, разделяющей зоны частиц и дырок с сильной дисперсией. Для спинонов с перпендикулярным направлением волнового вектора $\mathbf{q}_{\mathrm{HH}}$ реализуется совершенно плоский континуум спинонных возбуждений, что свидетельствует о полном отсутствии связи между спинонами в разных цепочках. Плоская дисперсия возбуждений между цепочками в нулевом магнитном поле наводит на мысль, что взаимодействие между цепочками при низких энергиях возбуждения заморожено, когда спинонная щель $\Delta_{\mathrm{S}} \neq 0$. Для спинонов с волновым вектором $\mathbf{q}_{\mathrm{L}}$ было установлено, что рост магнитного поля в интервале $0 \leq H \leq 30 \mathrm{kOe}$ сопровождается существенным изменением формы спектра возбуждений спинонов в $\mathrm{Yb}_{2} \mathrm{Pt}_{2} \mathrm{~Pb}$. Объясняется это тем, что рост магнитного поля приводит к изменению заселенности дисперсных зон спинонов с $S^{z}= \pm 1 / 2$ вследствие смещения химического потенциала $\mu$ в энергетической щели $\Delta_{\mathrm{S}}$. Зеемановское взаимодействие с магнитным полем понижает потенциал системы до значения $\mu=-g \mu_{\mathrm{B}} H S^{z}$, что может привести к захлопыванию щели в достаточно сильных полях. Получено, что величина потенциала, необходимого для закрытия щели и появления дырок при температурах $k_{\mathrm{B}} T<\Delta_{\mathrm{S}}$, равна $|\mu|=\Delta_{\mathrm{S}}=0.095 \mathrm{meV}$ и достигается с ростом поля до критического значения $H_{c 1}=5 \mathrm{kOe}$. В пределе относительно слабых полей, с ростом магнитного поля от нуля до значения $H_{c 1}$ вместо линейного увеличения был обнаружен скачок намагниченности. Было показано, что захлопывание щели при $H \geq H_{c 1}$ приводит к конфайнменту пар спинонов, который сопровождается появлением бесщелевой моды собственных продольных колебаний системы спиновых цепочек. В работе [9] исследовался конфайнмент низкоэнергетических возбуждений (спинонов) с дробными квантовыми числами в системе АФМ-цепочек спинов $S=1 / 2$, связанных слабыми обменными взаимодействиями в гейзенберговском $\mathrm{A} \Phi \mathrm{M} \mathrm{CaCu}_{2} \mathrm{O}_{3}$ с критической температурой АФМ упорядочения $T_{\mathrm{N}}=25 \mathrm{~K}$. Для состояний с пространственно ограниченным перемещением спинонов характерно, что спиноны в системе цепочек могут быть связаны вместе взаимодействием, сила которого возрастает с увеличением расстояния между ними. Одним из следствий этого является невозможность наблюдать такие возбуждения индивидуально. Авторы проводят аналогию конфайнмента спинонов в системе АФМ-цепочек спинов $S=1 / 2$ с подобными явлениями, которые были обнаружены ранее в физике элементарных частиц, где тяжелые частицы (барионы и мезоны) создаются вследствие конфайнмента кварков. Упрощенная картина конфайнмента спинонов наблюдается в цепочках с значительной Изингподобной анизотропией обменного взаимодействия, в которых основные состояния имеют конечный порядок Нееля. В этом случае спиноны играют роль доменных стенок, разделяющих два вырожденных состояния с противоположной намагниченностью. В случае цепочек с гейзенберговским обменом между спинами ситуация другая. Хотя в отдельной цепочке доменные стенки всегда создаются парами, отсутствует потеря энергии при их перемещениях относительно друг друга. Поэтому в таком случае в отдельной цепочке спиноны не связываются в пары спинов с $S=1 / 2$ сильным притяжением. В отличие от предела Изинга, в точке с нулевой анизотропией обменного взаимодействия между спинами существуют только два типа возбуждений цепочки: триплетные и синглетные. Возбуждения в отдельной цепочке (спиноны) со спином $S=1 / 2$ ограничены в перемещении даже бесконечно малой межцепочечной связью. Большинство исследованных ранее спиновых лестниц не обнаруживают это явление, так как сильное межцепочечное взаимодействие подавляет возбуждение спинонов при всех энергиях возбуждения. В работе [9] представлены результаты исследования рассеяния нейтронов для системы слабо связанных цепочек в спиновых лестницах в купрате $\mathrm{CaCu}_{2} \mathrm{O}_{3}$, который имеет 
орторомбическую симметрию Pтmn. Кристаллическая структура состоит из слоев $\mathrm{CuO}$, скирдованных вдоль направления $c$; спиновые цепочки лежат в плоскостях $a b$ вдоль направления $b$ и сдвинуты на половину элементарной ячейки вдоль оси $a$. Лестницы связаны между собой несколькими слабыми взаимодействиями. Внутри плоскостей $a b$ ионы $\mathrm{Cu}^{2+}$ на соседних лестницах связаны между собой через связи $\mathrm{Cu}-\mathrm{O}-\mathrm{Cu}$. Как и в других планарных купратах, в $\mathrm{CaCu}_{2} \mathrm{O}_{3}$ существует четырехспиновое обменное взаимодействие $J_{\text {cyclic, }}$ которое связывает четыре иона меди, формирующие прямоугольные площадки в плоскостях $a b$. Согласно расчетам других авторов, основные постоянные обмена равны: $J_{\text {leg }}=-147 \mathrm{meV} ; J_{\text {rung }}=-15 \mathrm{meV} ; J_{\text {cyclic }}=4 \mathrm{meV}$, где $J_{\text {leg }}, J_{\text {rung }}-$ постоянные продольного и поперечного обмена соответственно. Область между двумя спинонами (доменными стенками) в цепочке состоит из перевернутых спинов. Если такая цепочка связана антиферромагнитно с другой цепочкой в спиновой лестнице, то эти перевернутые спины имеют потерю энергии по сравнению с их параллельной ориентацией к спинам соседней цепочки. Эта потеря энергии, пропорциональная расстоянию между спинонами, приводит к их конфайнменту. В работе [9] было получено, что при высоких энергиях возбуждения система цепочек в лестницах имеет магнитные свойства, подобные несвязанным одиночным цепочкам. Сигнал рассеяния нейтронов в этом случае соответствует рассеянию, характерному для отдельной цепочки спинов $S=1 / 2$ с внутрицепочечной постоянной продольного обмена $J_{\text {leg }}=-162 \mathrm{meV}$. В то же время, при низких энергиях возбуждения цепочек нейтронами доминируют интегральные спиновые возбуждения, характерные для сильно связанных цепочек. Таким образом, слабо связанные лестницы в $\mathrm{CaCu}_{2} \mathrm{O}_{3}$ существенно отличаются от хорошо исследованных ранее сильно связанных спиновых лестницах, которые всегда находятся в режиме сильного конфайнмента и имеют возбуждения только в виде магнонов $(S=1)$. В работе [10] была исследована система Изинг-подобных слабо связанных антиферромагнитных XXZ-цепочек в $\mathrm{SrCo}_{2} \mathrm{~V}_{2} \mathrm{O}_{8}$ во внешнем магнитном поле с использованием метода терагерцевой спектроскопии с высоким разрешением. В области низких температур наблюдались серии возбуждений, с характерным зеемановским расщеплением во внешнем магнитном поле. Эти магнитные возбуждения были идентифицированы авторами как возбуждения пар спинонов, охваченных конфайнментом. Измеренный при температуре $6.5 \mathrm{~K}$ чуть выше $T_{\mathrm{N}}=5 \mathrm{~K}$ спектр поглощения при прохождении света через образец в интервале энергий $1.3-6 \mathrm{meV}$ содержит интенсивную линию $E_{1}$ поглощения света с частотой колебаний вблизи $\omega=0.35 \mathrm{THz}$ с последующей серией линий поглощения с энергиями $E_{2}, E_{3}, \ldots, E_{9}$, интенсивность которых резко падала до нуля с ростом частоты до $\omega=1.2 \mathrm{THz}$. Эти линии поглощения не наблюдались, когда магнитное поле $a c$ световой волны $h(\omega)$ было направлено параллельно направлению спинов в цепочках вдоль оси с кристаллической решетки. Это указывает на магнитную природу линий обнаруженного дискретного спектра поглощения света, вызванного возбуждением пар спинонов. Измеренная зависимость этих линий от внешнего магнитного поля $d c$ подтверждает этот вывод. В одиночной изинговской цепочке два спинона создаются переворотом спина. Спиноны, каждый из которых несет спин $-1 / 2$, могут свободно перемещаться вдоль цепочки за счет переворотов спинов без потери энергии. Это приводит к образованию сильно вырожденного первого возбужденного состояния с энергией $J$, равной внутрицепочечному обменному взаимодействию между ближайшими соседями. Конечная величина поперечной компоненты внутрицепочечного обменного взаимодействия снимает вырождение, что приводит к формированию континуума возбуждений двух спинонов, которые распространяются вдоль цепочки независимо. В присутствии слабых межцепочечных взаимодействий два спинона связаны между собой, так как их разделение будет разрушать межцепочечное обменное взаимодействие. Межцепочечное взаимодействие играет роль потенциала притяжения между спинонами $V(z)$, величина которого пропорциональна расстоянию $z$ между ними. Таким образом, причиной линейного конфайнмента спинонов в связанные пары является слабое межцепочечное взаимодействие. Это приводит к квантованию континуума возбуждений системы цепочек в $\mathrm{SrCo}_{2} \mathrm{~V}_{2} \mathrm{O}_{8}$ на дискретные уровни $E_{i}$ связанных пар спинонов, вызванному потенциалом $V(z)$. В работе [11] методом неупругого рассеяния нейтронов была исследована динамика возбуждения пар спинонов в Изинг-подобном антиферромагнетике $\mathrm{BaCo}_{2} \mathrm{~V}_{2} \mathrm{O}_{8}$ при температурах ниже $T_{\mathrm{N}} \approx 5.5 \mathrm{~K}$. Авторы отмечают, что исследование одномерного антиферромагнетика имеет особый интерес, так как квантовые флуктуации в одномерной системе спинов разрушают классический дальнодействующий неелевский порядок. При этом основное состояние становится разупорядоченным. Спектр возбуждений этого неупорядоченного основного состояния 1D-системы состоит из континуума многочисленных пар возбуждений со спином $S=1 / 2$ (спинонов), подобных доменным стенкам в изинговом магнетике. Однако одномерная система спинов может стать АФМ упорядоченной при очень низкой температуре при наличии даже малой связи между цепочками. Три возможных спиновых состояния $S= \pm 1,0$ для спинонных пар при этом трансформируются в две поперечные моды колебаний и третье - коллективное возбуждение, которое соответствует флуктуациям, параллельным направлению упорядоченного момента, то есть продольному типу колебаний. В работе [11] при температуре измерения $T=1.6 \mathrm{~K}$ ниже $T_{\mathrm{N}}$ обнаружен необычный спектр спиновых возбуждений, объясненный в терминах конфайнмента спинонов, вызванного межцепочечным взаимодействием. Эти возбуждения состоят из двух типов чередующихся серий с поперечной и продольной поляризацией соответственно. Подобные серии возбуждений изинговых спиновых цепочек наблюдались ранее в $\mathrm{CsCoCl}_{3}, \mathrm{CsCoBr}_{3}$ и $\mathrm{CoNb}_{2} \mathrm{O}_{6}$. Продольные колебания в цепочках соответствуют продольным флуктуациям 
упорядоченного момента. Анализ полученных экспериментальных результатов показал, что $\mathrm{BaCo}_{2} \mathrm{~V}_{2} \mathrm{O}_{8}$ с умеренной изинговой анизотропией и значительными межцепочечными взаимодействиями хорошо удовлетворяет условиям, необходимым для наблюдения долгоживущих продольных колебаний 1D-системы спинов. Суммируя полученные результаты анализа, авторы делают вывод, что возбуждения в $\mathrm{BaCo}_{2} \mathrm{~V}_{2} \mathrm{O}_{8}$ при температурах ниже $T_{\mathrm{N}}$ квантованы благодаря слабому межцепочечному взаимодействию и состоят из двух чередующихся серий поперечных колебаний и удивительно сильной продольной зеемановской лестницы. Предполагается, что стабилизация продольных мод становится возможной благодаря умеренной изинговой анизотропии, препятствующей их разложению на дискретные щелевые поперечные моды. В работе [12] методами упругого и неупругого рассеяния нейтронов при температуре $T=3.5 \mathrm{~K}$ были исследованы магнитные возбуждения в $\mathrm{BaCo}_{2} \mathrm{~V}_{2} \mathrm{O}_{8}$ в зависимости от напряженности поперечного $d c$ магнитного поля $H$ в интервале $0-10 \mathrm{~T}$, расположенного перпендикулярно к направлению системы АФМ-цепочек с Изингподобным обменом вдоль с оси кристаллической решетки. Проведенные ранее исследования влияния продольного магнитного поля на магнитные свойства $S=1 / 2$ $1 \mathrm{D}$-антиферромагнетика с Изинг-подобной анизотропией показали, что с ростом $H$ можно ожидать квантовый фазовый переход от упорядоченного состояния с дальнодействующим АФМ-порядком к неупорядоченной фазе типа спиновой жидкости. Это происходит благодаря развитию продольной спиновой корреляции, несоразмерной с кристаллической решеткой, с ростом напряженности магнитного поля. Конечная величина межцепочечных обменных взаимодействий приводит к появлению и росту фазы с дальнодействующей продольной волной спиновой плотности и изменениям ее несоразмерности с решеткой. Проводились также исследования влияния поперечных магнитных полей на фазовые превращения в подобных системах спиновых цепочек. Было показано, что поперечное внешнее магнитное поле также оказывает влияние на магнитный порядок в $\mathrm{BaCo}_{2} \mathrm{~V}_{2} \mathrm{O}_{8}$, например, подавляет его при значениях $H \approx 1 \mathrm{~T}$. Для нас представляют интерес результаты проведенного в [12] исследования влияния поперечных полей на спектр спинонов. Было установлено, что пиковые особенности серий спинонных возбуждений постепенно расщепляются с ростом внешнего магнитного поля. Было четко показано, что поведение спектра магнитных возбуждений в $\mathrm{BaCo}_{2} \mathrm{~V}_{2} \mathrm{O}_{8}$ в магнитном поле хорошо описывается на основе теории антиферромагнитной XXZ-цепочки спинов с $S=1 / 2$. Эта модель предсказывает, что динамический магнитный структурный фактор компоненты спина вдоль направления цепочки усиливается с ростом $H$, и появляются четко выраженные несоразмерные корреляции спинов. В работе [13] методом рассеяния нейтронов была обнаружена радикальная модификация квантовых возбуждений в $\mathrm{BaCo}_{2} \mathrm{~V}_{2} \mathrm{O}_{8}$ в поперечном магнитном поле при значениях $H$, превышающих критическую величину магнитного поля. Было показано, что этот результат вызван квантовым фазовым переходом между двумя различными видами солитонподобных топологических возбуждений. В более поздней работе [14] исследовался процесс конфайнмента спинонов в квазиодномерном анизотропном гейзенберговском антиферромагнетике $\mathrm{SrCo}_{2} \mathrm{~V}_{2} \mathrm{O}_{8}$ методом неупругого рассеяния нейтронов в широком интервале температур выше и ниже критической температуры $T_{\mathrm{N}}=5.2 \mathrm{~K}$ фазового перехода в АФМ-состояние. Наблюдаемый при температуре $T=6 \mathrm{~K}$ континуум спинонов в виде интенсивной пиковой особенности, размазанной в широком интервале энергий возбуждения спинонов, находится в хорошем соответствии с теоретическим предсказанием для поперечного динамического структурного фактора для гейзенберговской XXZ-цепочки при нулевой температуре. При температурах ниже $T_{\mathrm{N}}$ пары спинонов ограничены в перемещении, и наблюдаются две серии мезонподобных связанных состояний с продольной и поперечной поляризацией, соответствующие распространению возбуждения XXZ-цепочки нейтронами в продольном и поперечном направлении. Было найдено, что с понижением температуры до $T=1.5 \mathrm{~K}$ происходит трансформация широкой пиковой особенности в две серии узких резких пиков, ширина и интенсивность которых сильно зависят от условий возбуждения. Результаты нашего исследования $\mathrm{SmMnO}_{3+\delta}$ хорошо согласуются с картиной формирования бозонов в режиме конфайнмента спинонов.

\section{5. Заключение}

В настоящей работе были исследованы квантовые осцилляции температурной зависимости намагниченности $\mathrm{SmMnO}_{3+\delta}$ в магнитных полях $H=100$ и $350 \mathrm{Oe}, 1$ и $3.5 \mathrm{kOe}$ в интервале температур 4.2-12 K, вызванные квантованием Ландау спектра спинонных пар с коллинеарной ориентацией спинов $S=1 / 2$ в слабом мотовском диэлектрике. Осцилляции намагниченности $M(T)$ соответствуют квантованному калибровочным полем $b$ спектру низкоэнергетических возбуждений (спинонов) основного RVB-состояния щелевой $Z_{2}$ квантовой спиновой жидкости в виде периодических сдвоенных пиковых особенностей „сверхнамагниченности“, которые накладываются на экспоненциальное падение $M(T)$ при $T \rightarrow 0$. Континуум тепловых возбуждений „сверхнамагниченности“ спинонных пар в магнитном поле $H=100$ Ое делится на три перекрывающиеся зоны Ландау с энергиями $E_{n}(n=1,2,3)$ с дробными значениями фактора $v$ заполнения зон. Основной вклад в намагниченность вносит возбуждение пар спинонов в зоне с $n=3$ вблизи средней температуры $T_{\text {spinon }} \cong 8 \mathrm{~K}$ в виде симметричного интенсивного дублета „сверхнамагниченности“, состоящего из двух широких перекрывающихся пиков сверхнамагниченности. Это свидетельствует о существовании псевдощели $\Delta s \approx 0.4 \mathrm{meV}$ в континууме возбуждений спинонных пар в $\mathrm{SmMnO}_{3+\delta}$. Дублетное возбуждение спинонных пар происходит в непосредственной близости с температурой $T_{\mathrm{KT}}=T_{c} \cong 12 \mathrm{~K}$ топологического 
фазового перехода Костерлитца-Таулесса развязывания 2D-пар вихрь-антивихрь в когерентном сверхпроводящем состоянии, что указывает на кроссовер энергий возбуждения спинонных пар и свободных 2D-вихрей в $\mathrm{SmMnO}_{3+\delta}$. C ростом напряженности магнитного поля до значения $H=350$ Ое симметричная двугорбая пиковая особенность $M(T)$ с центром вблизи температуры $T_{\text {spinon }} \cong 8 \mathrm{~K}$ щелевого возбуждения спинонов с $S=1 / 2$ трансформируется в широкий асимметричный пик. При этом происходит сильное расширение спектра возбуждения спинонных пар в перекрывающихся зонах Ландау с номерами $n=1,2$ и 3. Этот результат свидетельствует о появлении „гигантских“ флуктуаций, возникающих в основном состоянии КСЖ, с ростом $H$, что приводит к размазыванию спектра низкоэнергетических спинонных возбуждений в зоне Ландау с номером $n=3$ по температуре. При дальнейшем росте поля до значения $H=1 \mathrm{kOe}$, широкий асимметричный пик „сверхнамагниченности“ трансформируется в симметричный синусоподобный пик, характерный для континуума возбуждений пар спинонов с сильной диперсией. Трансформация спектра спинонных пар с увеличением поля объясняется ростом поперечной компоненты внешнего магнитного поля, которое приводит к увеличению межцепочечного взаимодействия, уменышению псевдощели $\Delta_{s}$ и, соответственно, к росту плотности спинонных возбуждений в $\mathrm{AФМ} \mathrm{наноцепочек} \mathrm{спинов} \mathrm{в} \mathrm{SmMnO}_{3+\delta}$. В магнитном поле $H=3.5 \mathrm{kOe}$ спектр теплового возбуждения спинонов драматически изменяется: появляется новый тип квантования спектра спинонов в виде формировании в зонах Ландау $n=1,2$ и 3 пороговых особенностей намагниченности в виде узких ступенек (плато), соответствующих целочисленному заполнению спинонами трех зон Ландау с конечной щелью. С ростом температуры высота порогов и ширина ступенек растут. Появление новых квантовых осцилляций температурных зависимостей намагниченности в сильнополевом режиме измерений вызвано квантованием Ландау калибровочным полем плотного (несжимаемого) газа спинонов с дробным спином $S=1 / 2$, движущихся по круговым орбитам в направлении, поперечном направлению „магнитной компоненты $b$ калибровочного поля.

В настоящей работе также была исследована зависимость тепловых возбуждений жидкости Латтинжера в виде волн зарядовой и спиновой плотности при $T \leq 60 \mathrm{~K}$ от напряженности внешнего магнитного поля. В магнитном поле $H=100$ Ое в интервале температур $T_{1}<T<T_{2}$ шириной порядка $15 \mathrm{~K}$ формируется симметричная двухпиковая особенность „сверхнамагниченности“ образца в виде двух четко выраженных перекрывающихся пиков намагниченности $M(T)$ равной интенсивности вблизи температур 48 и $56 \mathrm{~K}$, которые вызваны тепловым возбуждением флуктуирующих квазиодномерных фрагментов развязанных волн зарядовой и спиновой плотности. С ростом напряженности магнитного поля до значения $H=1 \mathrm{kOe}$ картина резко изменилась: вместо симметричного дублета возбуждения развязанных волн зарядовой и спиновой плотности в интервале температур 44-58 K формируется ассиметричный пик „сверхнамагниченности“ в интервале температур 40-56 K, соответствующий возбуждению связанного состояния фрагментов волн зарядовой/спиновой плотности. При дальнейшем увеличении напряженности внешнего магнитного поля до значения $H=3.5 \mathrm{kOe}$ в интервале температур 32-60K возникает широкий синусоподобный пик температурной зависимости намагниченности $\mathrm{SmMnO}_{3+\delta}$, подобный возбуждению продольных спиновых волн (магнонов) в системе АФМ гейзенберговских спиновых цепочек. Таким образом, рост напряженности внешнего магнитного поля в высокотемпературном режиме измерений намагниченности $M(T)$ приводит к трансформации возбуждения смешанного состояния волн зарядовой и спиновой плотности в возбуждение только продольных 1D-волн спиновой плотности (магнонов). Согласно полевым зависимостям намагниченности $M(H)$, измеренных в различных режимах при температурах вблизи $T=0$, с ростом напряженности внешнего магнитного поля происходит квантовый фазовый переход КСЖ в состояние ЖЛ, индуцированный ростом напряженности внешнего магнитного поля. В результате этого перехода вблизи значения $H=0$ формируются пиковые особенности „сверхнамагниченности“ с различной степенью их перекрытия, характерные для возбуждения бесщелевых развязанных волн зарядовой и спиновой плотности в ЖЛ. Мы считаем, что такое поведение намагниченности $M(T)$ с ростом $H$ вызвано тем, что поперечная компонента внешнего магнитного поля закрывает спинонную щель $\Delta_{s}$ в $\mathrm{SmMnO}_{3+\delta}$ и стимулирует переход системы слабо связанных обменным взаимодействием зигзагообразных АФМ спиновых цепочек в состояние, подобное спиновой ЖЛ. Характерной особенностью этого состояния является пространственное разделение зарядовых и спиновых возбуждений. В этом квазиодномерном состоянии возбуждения многих частиц в виде 1D-волн зарядовой и спиновой плотности, которые подчиняются статистике Бозе, в основном заменяют элементарные возбуждения в виде спинонных пар.

\section{Конфликт интересов}

Авторы заявляют, что у них нет конфликта интересов.

\section{Список литературы}

[1] F.D.M. Haldane. Phys. Rev. Lett. 47, 25, 1840 (1981).

[2] T.L. Schmidt, A. Imambekov, L.I. Glazman. arXiv:1009.4708v2 [cond-mat.str-el]14 Dec 2010.

[3] S. Brazovskii, F. Matveenko, P. Nozieres. JETP Lett. 58, 10, 796 (1993).

[4] T. Vekua, S.I. Matveenko, G.V. Shlyapnikov. JETP Lett. 90, 4, 289 (2009).

[5] M. Rizzi, M. Polini, M.A. Cazalilla, M.R. Bakhtiari, M.P. Tosi, R. Fazio. Phys. Rev. B 77, 24, 245105 (2008).

[6] F.H.L. Essler, A.M. Tsvelik. Ann. Henri Poincaré C 4, Suppl. 2, S589 (2003). 
[7] D. Controzzi, F.H.L. Essler. Phys. Rev. B 66, 16, 165112 (2002).

[8] W.J. Gannon, I.A. Zaliznyak, L.S. Wu, A.E. Feiguin, A.M. Tsvelik, F. Demmel, Y. Qiu, J.R.D. Copley, M.S. Kim, M.C. Aronson. Nature Commun. 10, 1123 (2019). https://doi.org/10.1038/s41467-019-08715-y

[9] B. Lake, A.M. Tsvelik, S. Notbohm, D.A. Tennant, T.G. Perring, M. Reehuis, C. Sekar, G. Krabbes, B. Büchner. Nature Phys. 6, 1, 50 (2010).

[10] Z. Wang, M. Schmidt, A.K. Bera, A.T.M.N. Islam, B. Lake, A. Loidl, J. Deisenhofer. Phys. Rev. B 91, 14, 140404 (R) (2015).

[11] B. Grenier, S. Petit, V. Simonet, E. Canévet, L.-P. Regnault, S. Raymond, B. Canals, C. Berthier, P. Lejay. Phys. Rev. Lett. 114, 1, 017201 (2015).

[12] M. Matsuda, H. Onishi, A. Okutani, J. Ma, H. Agrawal, T. Hong, D.M. Pajerowski, J.R.D. Copley, K. Okunishi, M. Mori, S. Kimura, M. Hagiwara. Phys. Rev. B 96, 2, 024439 (2017).

[13] Q. Faure, S. Takayoshi, S. Petit, V. Simonet, S. Raymond, L.-P. Regnault, M. Boehm, J.S. White, M. Månsson, C. Rüegg, P. Lejay, B. Canals, T. Lorenz, S.C. Furuya, T. Giamarchi, B. Grenier. Nature Phys. 14, 7, 716 (2018).

[14] A.K. Bera, B. Lake, F.H.L. Essler, L. Vanderstraeten, C. Hubig, U. Schollwöck, A.T.M.N. Islam, A. Schneidewind, D.L. Quintero-Castro. Phys. Rev. B 96, 5, 054423 (2017).

[15] Ф.Н. Буханько, А.Ф. Буханько. ФТТ 63, 5, 639 (2021).

[16] S. Sachdev. arXiv: 0901.4103v6 (2009).

[17] O.I. Motrunich. Phys. Rev. B 72, 4, 045105 (2005).

[18] T. Senthil. Phys. Rev. B 78, 3, 035103 (2008).

[19] T. Senthil. Phys. Rev. B 78, 4, 045109 (2008).

[20] X.G. Wen. Lectures given in the Cargese Summer School of Strongly Correlated Electron Systems (1990).

[21] V. Kalmeyer, R.B. Laughlin. Phys. Rev. Lett. 59, 18, 2095 (1987).

[22] X.G. Wen, F. Wilczek, A. Zee. Phys. Rev. B 39, 16, 11413 (1989).

[23] R.B. Laughlin, Z. Zou. Phys. Rev. B 41, 1, 664 (1990).

[24] E. Mele. Phys. Rev. B 38, 13, 8940 (1988).

[25] K.V. Klitzing, G. Dorda, M. Pepper. Phys. Rev. Lett. 45, 6, 494 (1980).

[26] D.C. Tsui, H.L. Stormer, A.C. Gossard. Phys. Rev. Lett. 48, 22, 1559 (1982).

[27] R.B. Laughlin. Phys. Rev. B 23, 10, 5632 (1981).

[28] F.D.M. Haldane. Phys. Rev. Lett. 51, 7, 605 (1983).

[29] R.B. Laughlin. Phys. Rev. Lett. 50, 18, 1395 (1983).

[30] F.D.M. Haldane, Y.-S. Wu. Phys. Rev. Lett. 55, 26, 2887 (1985).

[31] F.D.M. Haldane. Phys. Rev. Lett. 67, 8, 937 (1991).

[32] F. Wilczek. Phys. Rev. Lett. 49, 14, 957 (1982).

[33] B.I. Halperin. Phys. Rev. Lett. 52, 18, 1583 (1984).

[34] R.B. Laughlin. Phys. Rev. Lett. 60, 25, 2677 (1988).

[35] G. Misguich, T. Jolicoeur, S.M. Girvin. Phys. Rev. Lett. 87, 9, 097203 (2001).

[36] K. Kumar, K. Sun, E. Fradkin. Phys. Rev. B 90, 17, 174409 (2014).

[37] K. Kumar, K. Sun, E. Fradkin. Phys. Rev. B 92, 9, 094433 (2015).

Редактор Е.В. Толстлкова 